\title{
HIV testing uptake and yield among sexual contacts of HIV-positive men who have sex with men in Zhejiang Province, China, 2014-2016: a cross-sectional pilot study of a choice-based partner tracing and testing package
}

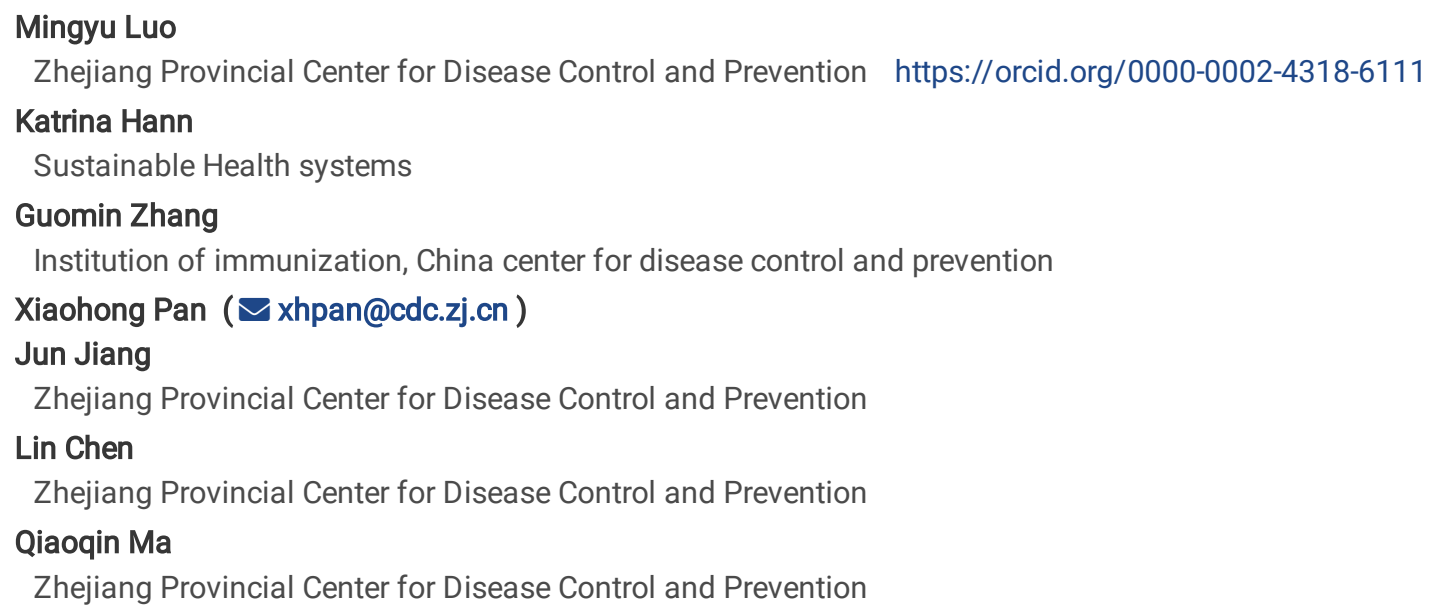

Research article

Keywords: HIV testing preferences; HIV tracing and testing; MSM; partner notification service

Posted Date: July 5th, 2019

DOI: https://doi.org/10.21203/rs.2.11001/v1

License: @) (i) This work is licensed under a Creative Commons Attribution 4.0 International License. Read Full License 


\section{Abstract}

Background Measures to effectively expand tracing and testing to identify undiagnosed HIV infections are significant for the control of HIV/AIDS epidemic among men who have sex with men (MSM). We piloted a combination tracing and testing package aimed at improving contact tracing and uptake and yield of HIV testing for sexual contacts of newly diagnosed HIV positive MSM. We describe the package components and outcomes. Methods The choice-based tracing and testing package was piloted in Hangzhou and Ningbo cities, June 2014 to June 2016. The package adopted four modes, including couples HIV counselling and testing (CHCT), information-driven assisted partner notification (IDAPN), peer-assisted HIV self-testing (HIVST), and patient referral. If sexual contacts tested positive, they were invited to enroll in the pilot. Contact tracing continued through selected modes until inability to contact, refusal to test, or positive test results exhausted all contacts. Socio-demographic factors, sexual behaviors between HIV-positive MSM and their sexual contacts were collected, as well as tracing and testing outcomes of each mode. Results Among 2,495 newly diagnosed HIV-positive MSM, 446(18\%) were enrolled as index cases (ICs) through two rounds of contact tracing. The ICs disclosed a total of 4,716 sexual contacts, of whom $548(12 \%)$ were reachable. The pilot resulted in a testing uptake of $87 \%(478 / 548)$ and a yield of $16 \%(74 / 478)$ among sexual contacts. Logistic regression analysis showed that the odds of a reachable sexual contact enrolled in information-driven mode taking an HIV test were $90 \%$ more than that of one enrolled in patient referral $(95 \% \mathrm{Cl}: 0.8,4.4)$. Conclusions Choice-based tracing and testing package is feasible in expanding HIV-testing uptake among sexual contacts of HIV-positive MSM and are feasible for case-finding among a high-risk population. IDAPN may be an acceptable option to reach sexual contacts for whom limited contact information is available.

\section{Background}

Men who have sex with men(MSM) are at substantial risk of HIV/AIDS infection in China, with an almost six-fold increase of newly diagnosed infections from 2010 to 2016, and a 7.75\% prevalence of HIV infection in MSM in 2016(similar to European countries). [1,2] Failure to detect infection is a significant driver of the HIV epidemic amongst MSM. [3,4] Globally, HIV testing rates amongst MSM range from 50\% to 70\%, [5-7] indicating a large proportion of undiagnosed HIV infections.

HIV tracing and testing approaches are essential to identify undiagnosed infections and prevent further transmission among sexual contacts of HIV-positive MSM. [8,9] The Chinese government has advocated for an increase in HIV testing among high risk populations since 2012,[10] which resulted in the introduction of voluntary counselling and testing clinics (VCT clinics) services among other services. [11] The introduction of VCTs in China allowed for MSM to visit testing sites and find out their infection status through passive case finding, in which MSM initiate to take HIV testing, even with non-government organization(NGO)'s outreach efforts.[12] The rate of HIV testing amongst MSM increased from 50\% to $65 \%$ from 2011 to 2015. [1] However, with 25\% of HIV infections amongst MSM remaining undiagnosed, [13] active case finding approaches, in which health care providers initiate to target this key population may be a significant step towards addressing this testing gap.

Emerging evidence points to acceptability, feasibility, and transferability of existing intervention models for active case finding and referral. WHO defines options for assisted partner referral options, in which trained health care providers assist clients to disclose their partners' potential exposure to HIV infection and refer their partners to HIV testing services (HTS).

In assisted partner notification services, information-based tracing and testing mode utilizing social network applications may provide adequate convenience and privacy protection by providing a direct communication line for health providers and contacts. [14,15] By tracing sexual contacts using social media account identifiers, such an approach avoids contact between the HIV positive MSM and their sexual contacts. [16] Social media point-to-point interventions, in which the healthcare provider communicates directly to the contact, have been shown as feasible in reaching MSM in previous studies. [14] This mode is effective to identify high numbers of HIV-positive individuals in need of treatment, and is cost-effective on locating and contacting partners. [17]

Couples' HIV counselling and testing (CHCT) is another sub type of assisted partner notification, in which couples test, share results and receive interventions together, may be an acceptable approach among MSM and their sexual contacts. $[18,19]$ However, we need more evidence on contact tracing of HIV positive MSM.

Assisted HIV Self-Testing (HIVST) is in which individuals who are self-testing for HIV receive an in-person demonstration from a trained provider or peer before or during HIVST. HIVST is an acceptable strategy that can increasing testing uptake among high risk populations who are less likely to access testing. $[17,20,21]$ In HIVST, oral HIV self-testing may be a complementary method for screening sexual contacts who fear disclosure of HIV status, however, it hasn't consistently been found to be acceptable. [22,23]

In practice, active and passive contact tracing are complementary. Care providers may offer more than one testing mode for clients, allowing him to select an option which reduces barriers to testing specific to the individual. Sharma et al. [22] explored MSM attitudes and usage preference towards six different HIV testing modes presented collectively. Such results were identified low acceptability for one intervention option and specific intervention options of preference for different sub-groups. [24]

Page $2 / 13$ 
In China, rare evidence remains on choice-based partner tracing and testing for tracing of sexual social contacts and testing uptake of HIV positive MSM. Such evidence is essential for the HIV prevention and control program to establish guidelines on active case finding strategies targeting special populations, such as MSM and their sexual contacts. In this study, we describe a pilot study of a choice-based partner tracing and testing program aimed at analyzing uptake and infection status of HIV testing for sexual contacts of newly diagnosed HIV positive MSM, its intervention components, and outcomes in Zhejiang Province, China.

\section{Methods}

\section{Selection and Description of Participants}

We defined the study population as newly diagnosed HIV positive MSM and their sexual contacts who agreed to participate in the partner tracing and HIV testing program in VCT clinic study sites in Hangzhou and Ningbo cities, between June 2014 through June 2016 . We defined the inclusion criteria for index cases (ICS) as male, age of 18 years or above, having been newly diagnosed as HIV positive, having disclosed male sexual contacts (including oral intercourse or anal intercourse) to VCT clinical staff in pre- or post-testing counselling at time of diagnosis, and having agreed to participate in partner tracing and testing program. We excluded any potential participants with a cognitive impairment, severe AIDS complication or other illnesses requiring hospitalization, or other disability, as determined by clinical staff at the VCT clinic. We defined inclusion criteria for sexual contacts as reported sexual contacts of ICs in post-testing counselling at time of HIV testing, but excluded those with cognitive impairment, severe AIDS complication or other illness requiring hospitalization, or disability, as determined by clinical staff at VCT clinic. We defined reachable sexual contacts as those can be contacted by ICs or VCT clinical staff via facing each other or targeted by contact information (geographical location or social software accounts/phone number). We did not include an exclusion criterion with regards to age for the sexual contacts in order to identify any high social risk between adult IC and minor sexual contacts. However, HIV positive sexual contacts under 18 years old were interviewed with their legal guardians' consent and presence.

\section{Technical Information}

Study design

We undertook a cross-sectional study describing a pilot of a choice-based partner tracing and testing program offering a choice of four modes to analyze HIV testing uptake and identification of undiagnosed infections among sexual contacts of HIV positive MSM.

Study setting

The choice-based partner tracing and testing program aims to increase HIV testing uptake and yield amongst sexual contacts of newly diagnosed HIV positive MSM by offering a choice in modality of partner tracing and testing package. The package targets sexual contacts of newly diagnosed HIV men disclosed in post-testing counselling at VCT clinics. VCT clinical staff introduce the package to potential participants post-testing and invite the MSM to enroll as an IC. ICs are introduced to a choice of four modes for partner tracing and HIV testing, and they need to commit the preferred mode when they get enrolled.

The enrollment proceeds to the next round if tested and positive sexual contacts consent and be enrolled as ICs. The process ended when no positive is identified among sexual contact, no sexual contact is reachable, or no positive sexual contact consent and be enrolled as ICs.

The partner tracing and HIV testing package includes: 1) Couples HIV counselling and testing (CHCT): ICs brought sexual contacts to VCT clinics, in which they take HIV testing and receive counselling together; 2) Information-driven assisted partner notification (IDAPN): ICs provide social media or phone contact information of sexual contacts. The VCT staff then contact the sexual contact through these communication tools and promote he/she to undertake HIV testing, without disclosure ICs' identity; 3) Assisted HIV self-testing (HIVST): The VCT staff trains the ICs how to use an oral rapid HIV testing kit and provide testing kits; the IC then train and provide the sexual contacts with the oral rapid HIV testing kit, and ICs return testing kits to the clinic; 4) Patient referral: ICs are responsible to notify sexual contacts and promote them to take HIV-testing by themselves. An additional figure file shows the four modes in more detail [see Additional file 1].

The partner tracing and HIV testing pilot was implemented in all 23 Centers for Disease Control and Prevention (CDC) VCT clinic sites in Hangzhou and Ningbo cities from June 2014 to June 2016. VCT clinical staff received structured training (duration 2 days) by Zhejiang Provincial CDC. The training included procedure of partner tracing and HIV testing package, including how to elicit ICs and their sexual contacts, and privacy concerns. All HIV testing procedures and follow up care follow National Guideline for Detection of HIV/AIDS of China.[25] We utilize the RECORD guidelines to report on the study. [26]

Data sources and variables

Page $3 / 13$ 
Data of the ICs and sexual contacts were collected by VCT clinical staff, who received training on data collection and research ethics. VCT clinical staff administered a short questionnaire on sexual contacts and risk behaviors with ICs at VCT sites besides routine counselling after HIV diagnosis.

The questionnaire included questions related to IC demographics; sexual contacts' gender; current status of sexual relationship (currently in a relationship, not currently in a relationship, unsure); types of sexual relationship; frequency of sexual contact in the previous 6 months for stable relationships, or ever for unstable; and condom use in the previous 6 months. Stable relationships were defined as either a reported stable, non-commercial contacts, either male or female spouse. Unstable relationships were defined as either a reported commercial male contact; casual, non-commercial male contact, or an unmarried female contact; Modes of partner tracing and testing package selected by ICs (Patient referral and CHCT; Information-driven assisted partner notification (IDAPN); Assisted HIV-self testing (HIVST); Patient referral and VCT). Data on these were collected from ICs.

Data on sexual contact HIV testing uptake and testing outcome were completed from laboratory records.

The research team extracted data from database provided by the VCT staff every three months.

\section{Statistics}

We utilized EpiData 3.0 [Epidata.dk] for data entry and SPSS 20.0[IBM Corp.] to conduct quantitative analyses. We presented ICs and sexual contacts' characteristics and compared mode participation using frequencies and proportions for categorical variables. We calculated the mode preference of ICs by assigning a preference value of one to each IC and proportionally assigning this value across his selection for each sexual contact.

We compared HIV testing uptake (Tested sexual contacts/Reachable sexual contacts) and yield (HIV-positive sexual contacts/Tested sexual contacts) of sexual contacts across different modes, and presented the estimated effect size using odds ratios. We assigned statistical significance at $p$-values less than 0.05 .

\section{Results}

Between June 2014 and June 2016, 2,495 newly diagnosed HIV-positive MSM from VCT clinics in Hangzhou and Ningbo were invited to participate in the study. 435 consented and enrolled as ICs, resulting in an enrollment of 17\%. Following the program guidelines, the pilot resulted in two rounds of contact tracing and 4176 disclosed sexual contacts. Of those disclosed, 548(13\%) were reachable. First-round ICs disclosed 4,116 sexual contacts; 537 were reachable. Of those reached, 467 accepted HIV testing, 65 tested positive and 11 consented to enroll as second-round ICs. These second-round ICs disclosed 60 sexual contacts, 11 of whom were reachable. All reachable second- round contacts accepted HIV testing, which resulted in nine HIV-positive test results. An additional figure file shows this in more detail [see Additional file 2] Overall, the mean number to successfully recruit a reachable sexual contact for HIV testing was 1.1(548/478), and 6.5(478/74) to identify a positive.

In total, $446 \mathrm{ICs}$, both first- and second-round, enrolled in the pilot, with a mean age of 31.5(SD=9.6) years old. The majority of ICs had a college education or above $(212,48 \%)$, lived in the local county of the VCT $(225,52 \%)$, and disclosed six or more sexual contacts $(239,54 \%)$. The two rounds of tracing and testing in the pilot resulted in 4,176 disclosed and 548 reached sexual contacts. (Figure 1) Mean age of sexual contacts was $30.7(S D=8.9)$ years old, and most were male $(460,84 \%)$. Nearly half of reachable sexual contacts $(267,49 \%)$ were currently in a sexual relationship with ICs. Among reachable sexual contacts in a stable relationship with the IC, over half $(179,60 \%)$ had sexual contact with ICs less than once per week. Among those in casual relationships, almost half $(110,45 \%)$ had sexual contact with ICs a total of two to five times. Only about one third of reachable sexual contacts (34.7\%) used condoms with ICs consistently in the past six months. (Table 1)

ICs selected the patient referral and CHCT mode most frequently $(163,37 \%)$ and HIVST $(13,3 \%)$ the least (see Table 2$).$

HIV testing uptake among all reachable sexual contacts was $87 \%$ and varied between $82-94 \%$ across the selected modes. Sexual contacts reached through the IDAPN mode showed the highest testing uptake (94\%).

HIV testing yield among all tested sexual contacts was 16\%. Among the selected testing and tracing modes, patient referral yielded $18 \%$, CHCT $17 \%$, and IDAPN 7\%. (see Table 2)

We found the odds of a reachable sexual contact traced and tested through CHCT taking an HIV test were $40 \%$ lower than one traced and tested through patient referral $(95 \% \mathrm{Cl}: 0.3,1.0)$. The odds of a reachable sexual contact traced and tested through IDAPN taking an HIV test were $90 \%$ more than that of one traced through patient referral $(95 \% \mathrm{Cl}: 0.8,4.4)$. The odds of a reachable sexual contact traced and tested through $\mathrm{CHCT}$ testing HIV-positive were $10 \%$ more than that of one traced through patient referral $(95 \% \mathrm{Cl}: 0.6,1.9)$. Conversely, the odds of a 
reachable sexual contact traced and tested in IDAPN testing HIV-positive were $70 \%$ lower than that of one traced through patient referral $(95 \% \mathrm{Cl}: 0.1,0.6)$. (Table 3)

In different type of sexual relationship with sexual contacts, $43 \%$ (93/219) of stable same-sex contacts and 55.1\% (43/78) opposite-sex spouse were designated to CHCT; $40 \%$ (95/236) of casual same-sex contacts were designated to IDAPN. In casual, non-commercial same-sex relationship, those traced and tested through IDAPN taking an HIV test presented higher proportion than those traced and tested through CHCT taking an HIV test. An additional table file shows this in more detail [see Additional file 3]

\section{Discussion}

We presented results from a pilot of a combination partner tracing and HIV-testing program to expand HIV testing uptake for sexual contacts of HIV-positive MSM. Our results showed that this package is a feasible approach in this setting, which helps target and locate high risk population.

We found a high proportion (87\%) of reachable, sexual contacts were successfully tested, which is higher than a previous study in eastern China (23\%) and similar to one in Hangzhou and Kunming(in Yunnan province, southwest of China) (85\%).[27,28] The mean number to successfully recruit a reachable sexual contact for HIV testing was 1.1(548/478). This suggests potential advantages of this partner tracing and testing package in expanding HIV testing uptake among reachable sexual contacts of HIV-positive MSM and points to needed further research on effectiveness of testing uptake.

We found a higher HIV testing yield (16\%) amongst tested sexual contacts of HIV positive MSM than the general population HIV prevalence in Zhejiang province (estimated at 0.04\% [13] ) and general population passive tracing (VCT) testing yield of its cities (1.30\% in Hangzhou [29] ) We expected these results due to MSM higher risk behaviors. [7,30,31] Our testing yield result was also higher than that of MSM in a city in Zhejiang province contacted through passive tracing (VCT; 4.19\% in Lishui, 2008-2015[32] ). Previous study also proved that partner tracing and testing may be more effective in identifying undiagnosed infections than other HIV testing policies.[28] Such findings suggest a higher efficiency of integrating active case finding strategies with passive in order to target contacts of MSM in this setting.

Our results showed variation in testing uptake and yield across the different tracing and testing modes. In the CHCT mode, reachable sexual contacts presented the lowest rate of HIV-testing uptake and less odds of uptake than those in the patient referral mode. As it requires couples take HIV testing and counselling together,[18] fear of disclosure or discrimination may be barriers to selection of this mode. [33] Our results of CHCT uptake (82\%) are similar to a previous study in China showed high willingness to receive CHCT (86\%).[34] However, this mode resulted in effective case finding as compared to the others, which may indicate it may be more acceptable for same-sex couples who are in or seeking committed relationships. [35] Our results also showed that CHCT is more acceptable among stable contacts. This is more likely to engage in unprotected anal sex, thus at higher risk of HIV transmission, [36] which may contribute to our results of high yield for those who did get testing in this mode. Therefore, CHCT may be feasible to identify undiagnosed infections and target stable sexual contacts.

The information-driven partner notification presented the highest HIV-testing uptake. Although this difference was not statistically significant, it is in line with previous studies which showed acceptability of similar methods.[15] Because this mode did not require direct contact between ICs and sexual contacts, which may be effective to target sexual contacts with limited contact information (such as only having social media contact information) and protect privacy of both sides. Information-driven partner notification may result in high testing uptake when ICs do not have the intention or ability to notify sexual contacts.[21] However, in our study, case finding in the information-driven partner notification was not as effective as that for $\mathrm{CHCT}$ and patient referral. This may reflect the fact that the choice of information-driven mode may indicate a less intimate relationship.

Previous studies showed that oral HIV self-testing can be feasible and effective for MSM and their sexual contacts. [37] However, our results indicate that fewer participants chose this mode for their sexual contacts. As ICs needed to learn how to use oral HIV self-testing kits and teach this skill to their sexual contacts, the overly complex instructional materials and procedure may have restricted ICs' intention to bring kits to their partners.[38] Further studies and practical application are needed to identify whether oral HIV self-testing is feasible on expanding HIVtesting in sexual contacts of HIV-positive MSM in this setting. Results showed that Patient referral is still an important component in expanding HIV testing and case-identification amongst MSM and should continue to be an option amongst multi-modal HIV-testing uptake programs.

There are some limitations to this research. As we utilized self-reported data from ICS, which might bring information bias for characteristics of sexual behavior between ICs and sexual contacts. In addition, enrollment in the study was limited, with 435 of 2508 total newly diagnosed HIV positive MSM agreeing to participate, and only 13\% $(548 / 4,176)$ of disclosed contacts of ICs were traceable. However, the package has effectively increased HIV testing uptake among HIV positive MSM who are enrolled as ICs and reachable sexual contacts. The findings are still significant as it still gives an insight into what needs to be done. 


\section{Conclusions}

In conclusion, partner tracing and testing package including $\mathrm{CHCT}$, information-driven partner notification may have the potential to play important role in expanding HIV-testing uptake among sexual contacts of HIV-positive MSM and are effective for case-finding among a highrisk population. An information-driven partner notification may be an acceptable option to reach sexual contacts for whom limited contact information is available. However, further study is needed on the feasibility of oral HIV self-testing amongst sexual contacts of MSM.

\section{Abbreviations}

HIV: Human Immunodeficiency Virus

AIDS: Acquired Immune Deficiency Syndrome

MSM: Men who have sex with men

VCT: Voluntary Counselling and Testing Clinics

NGO: Non-Government Organization

HTS: HIV testing services

CHCT: Couples' HIV counselling and testing

HIVST: Assisted HIV Self-Testing

IC: index case

IDAPN: Information-driven assisted partner notification

\section{Declarations}

Ethics approval and consent to participate

The study was approved by Institutional Review Boards of Zhejiang Provincial Center for Disease Prevention and Control (N0.: 2013-001) and all participants provided informed consent. Parent consent and informed assent was obtained for persons under 18 years.

Consent for publication

Participants' consent form is available.

Availability of data and material

The datasets generated and/or analysed during the current study are not publicly available due the datasets contained information of people with HIV/AIDS, which is sensitive.

Competing interests

None declared.

Funding

Funding for the costs of publication in an open-access, peer-reviewed journal was supported by Key Project on Social Development among S\&T Major Project of Zhejiang Province, China (2013C03047-1), Zhejiang Provincial Medicine Science and Technology Plan(2015PYA004), National Science and Technology Major Project of China (2017ZX10201101), The Training Project of Young Scientific and Technological Innovative Talents of Zhejiang Provincial Center of Disease Control and Prevention.

Authors' contributions

$\mathrm{PX}, \mathrm{XS}$ and MQ were involved in the study design and conduction; CL and JJ were involved in the conduction and supervised the data collection; $\mathrm{LM}, \mathrm{KH}$, and ZG analyzed and interpreted the data and wrote the first draft of the manuscript. All authors reviewed the draft manuscript content critically and approved of the final version. 
This research was conducted through the Structured Operational Research and Training Initiative (SORT-IT), a global partnership coordinated by the Special Programme for Research and Training in Tropical Diseases at the World Health Organization (WHO/TDR). The training model is based on a course developed jointly by the International Union Against Tuberculosis and Lung Disease (The Union) and Médecins Sans Frontières (MSF). The Specific SORT-IT programme which resulted in this publication was implemented by: Médecins Sans Frontières, Brussels Operational Centre, Luxembourg and the China Centre for Disease Control \& Prevention. Mentorship and the coordination/facilitation of this SORT-IT workshop were provided through the University of Washington, Department of Global Health, USA; AMPATH, Eldoret, Kenya; Sustainable Health Systems, Sierra Leone; Universidad Pontificia Bolivariana, Columbia; Global AIDS Interfaith Alliance, USA; Centre for Operational Research, The Union, Paris, France; and the China Centre for Disease Control \& Prevention. Thanks to all CDC staff in Hangzhou and Ningbo cities.

\section{References}

1. UNAIDS. HIV prevalence of Men who have sex with men. http://www.aidsinfoonline.org/kpatlas/\#/home.

2. NCAIDS, NCSTD CC. Update on the AIDS/STD epidemic in China in December,2016. Chinese J AIDS STD. 2017; $23: 93-5$.

3. Wong NS, Wong KH, Lee MP, Tsang OTY, Chan DPC, Lee SS. Estimation of the undiagnosed intervals of HIV-infected individuals by a modified back-calculation method for reconstructing the epidemic curves. PLoS One. 2016;11:1-15.

4. Zou H, Hu N, Xin Q, Beck J. HIV testing among men who have sex with men in china: A systematic review and meta-analysis. AIDS Behav. $2012 ; 16: 1717-28$

5. Brito AM, Kendall C, Kerr L, Mota RMS, Guimarães MDC, Dourado I, et al. Factors associated with low levels of HIV testing among men who have sex with men (MSM) in Brazil. PLoS One. 2015; 10:1-13.

6. Carvalho C, Fuertes R, Lucas R, Martins A, Campos MJ, Mendão L, et al. HIV testing among Portuguese men who have sex with men results from the European MSM Internet Survey (EMIS). Hiv Med. 2013; 14:15-8.

7. Li R, Wang H, Pan X, Ma Q, Chen L, Zhou X, et al. Prevalence of condomless anal intercourse and recent HIV testing and their associated factors among men who have sex with men in Hangzhou, China: A respondent-driven sampling survey. PLoS One. 2017; 12:1-18.

8. McDaid LM, Hart GJ. Increased HIV testing and reduced undiagnosed infection among gay men in Scotland, 2005-8: Support for the optout testing policy? Sex Transm Infect. 2011; 87:221-4.

9. Ministry of Health, PRC, UNAIDS W. Estimation of HIV epidemic in China,2011. Chinese J AIDS STD. 2012; 18:1-5.

10. Council GO of the S. Circular of the General Office of the State Council on the Issue of the 12th Five-Year Plan of Action to Combat and Combat AIDS in China. 2012. http://www.gov.cn/zwgk/2012-02/29/content_2079097.htm.

11. Guo W, Song AJ, Wang B. HIV/AIDS related high-risk behaviors among men who have sex with men (MSM) in Langfang,Hebei province. Chinese J Aids Std. 2009.

12. Xu Zuhui, Li Feng, Wang Ni, Zhang Liping, Luo Mei FY. Impact of China-Gates program on NGO participating in the mobilization of HIV testing in MSM in seven cities. Chinese J AIDS STD. 2013; 19:406-8. doi:10.13419/j.cnki.aids.2013.06.011.

13. Xu Yun, Pan Xiaohong, Zheng Jinlei, Jiang Jun, He Lin, Chen Weiyong YJ. Estimation of HIV epidemic in Zhejiang province,2015. Dis Surveiliance. 2017; 32:118-21.

14. Phillips G, Magnus M, Kuo I, Rawls A, Peterson J, Jia Y, et al. Use of geosocial networking (GSN) mobile phone applications to find men for sex by men who have sex with men (MSM) in Washington, DC. AIDS Behav. 2014; 18:1630-7.

15. D. L, A. C, T.P. L, C.M. M, W. Y. Reaching suburban men who have sex with men for STD and HIV services through online social networking outreach: A public health approach. J Acquir Immune Defic Syndr. 2016;72:73-8.

http://journals.Iww.com/jaids/pages/default.aspx\%0Ahttp://ovidsp.ovid.com/ovidweb.cgi?

$T=J S \& P A G E=$ reference\&D=emed18b\&NEWS=N\&AN=610638419.

16. Kirby T, Thornber-Dunwell M. Phone apps could help promote sexual health in MSM. Lancet. 2014; 384:1415. doi:10.1016/S01406736(14)61849-3.

17. World Health Organization. Guidelines on HIV self-testing and partner notification: supplement to consolidated guidelines on HIV testing services. 2016.

18. Wagenaar BH, Christiansen-Lindquist L, Khosropour C, Salazar LF, Benbow N, Prachand N, et al. Willingness of us men who have sex with men (MSM) to participate in couples HIV voluntary counseling and testing (CVCT). PLoS One. 2012; 7:1-8.

19. Mitchell JW. Gay male couples' attitudes toward using couples-based voluntary HIV counseling and testing. Arch Sex Behav. 2014; 43:161-71. doi:10.1007/s10508-013-0211-0. 
20. Dalal S, Johnson C, Fonner V, Kennedy CE, Siegfried N, Figueroa C, et al. Improving HIV test uptake and case finding with assisted partner notification services. Aids. 2017; 31:1867-76.

21. van Aar F, Schreuder I, van Weert Y, Spijker R, Götz H, Op de Coul E, et al. Current practices of partner notification among MSM with HIV, gonorrhoea and syphilis in the Netherlands: An urgent need for improvement. BMC Infect Dis. 2012;12.

22. Katz DA, Golden MR, Hughes JP, Farquhar C, Stekler JD. Acceptability and ease of use of home self-testing for HIV among men who have sex with men. http://www.hivforum.org/storage/documents/_2012NationalSummit_Posting/a_044_katz.pdf. 2012;2:50. http://www.hivforum.org/storage/documents/_2012NationalSummit_Posting/a_044_katz.pdf.

23. Huo Junli, Shi Yuhua, LiJunjie, Cui Wenqing, Zhangxiujie, Hu Yi, Su Xingfang J. Factors associated with willingness to accept and use oral fluid HIV rapid testing. Chinese J Dis Control Prev. 2015; 19:1260-4.

24. Sharma A, Stephenson RB, White D, Sullivan PS. Acceptability and intended usage preferences for six HIV testing options among internetusing men who have sex with men. Springerplus. 2014; 3:1-10.

25. China CDC. National Guideline for Detection of HIV/AIDS. 2015.

26. Benchimol El, Smeeth L, Guttmann A, Harron K, Moher D, Petersen I, et al. The REporting of studies Conducted using Observational Routinely-collected health Data (RECORD) statement. PLoS Med. 2015;12: e1001885.

27. Lin H, He N, Ding Y, Qiu D, Zhu W, Liu X, et al. Tracing sexual contacts of HIV-infected individuals in a rural prefecture, Eastern China. BMC Public Health. 2012;12.

28. Fu X, Qi J, Hu Y, Pan X, Li Y, Liu H, et al. Partner notification in cooperation with community-based organizations among HIV-positive men who have sex with men in two Chinese cities. Int J STD AIDS. 2016; 27:821-31. doi:10.1177/0956462416648827.

29. Li Xiting, Luo Yan, Cheng Jie, Zhang Xingliang, Jin Dandan, Jin Jie, Ding Jianming LX. Dynamic trend of voluntary counsel and test for HIV in Hangzhou, Zhejiang,2012-2014. Dis Surveiliance. 2015; 30:742-5.

30. Zhang J, Guo Z, Pan X, Zhang W, Yang J, Ding X, et al. Highlighting the crucial role of Hangzhou in HIV-1 transmission among men who have sex with men in Zhejiang, China. Sci Rep. 2017; 7:1-11. doi:10.1038/s41598-017-14108-2.

31. X. P, J. J, Q. M, J. Z, J. Y, W. C, et al. Outbreak of HIV infection linked to nosocomial transmission, China, 2016-2017. Emerg Infect Dis. 2018; 24:2141-9. doi: http://dx.doi.org/10.3201/eid2412.180117.

32. Tao Tao, Lei Yongliang, Qiu Liyang, Chen Xiaolei SW. Analysis of epidemic of AIDS among men who have sex with men in Lishui city,20082015. Chinese J Public Heal Manag. 2016; 32:887-9.

33. Li X, Lu H, Raymond HF, Sun Y, Jia Y, He X, et al. Untested and undiagnosed: Barriers to HIV testing among men who have sex with men, Beijing, China. Sex Transm Infect. 2012; 88:187-93.

34. Wei C, Muessig KE, Bien C, Yang L, Meng R, Han L, et al. Strategies for promoting HIV testing uptake: Willingness to receive couple-based and collective HIV testing among a cross-sectional online sample of men who have sex with men in China. Sex Transm Infect. 2014; 90:469-74.

35. Stephenson R, Sullivan PS, Salazar LF, Gratzer B, Allen S, Seelbach E. Attitudes towards couples-based HIV testing among MSM in three US cities. AIDS Behav. 2011;15 SUPPL. 1:1-12.

36. Marks G, Crepaz N, Senterfitt JW, Janssen RS. Meta-analysis of high-risk sexual behavior in persons aware and unaware they are infected with HIV in the United States: Implications for HIV prevention programs. J Acquir Immune Defic Syndr. 2005; 39:446-53.

37. Yu Maohe, Guo Yan, Zhou Ning, Dong Xiaoyue, Xu Peng, Xu Jie, Gao Yongjun, Li Zhijun YJ. Application of HIV oral rapid test among MSM in a gay bathhouse. Chinese J Dis Control Prev. 2014; 18:1229-31.

38. Estem KS, Catania J, Klausner JD. HIV Self-Testing: A Review of Current Implementation and Fidelity. Curr HIV/AIDS Rep. 2016;13:107-15.

\section{Tables}

Table 1. Newly diagnosed HIV-positive men who have sex with men enrolled in a partner tracing and HIV testing program, June 2014 through June 2016, in Hangzhou and Ningbo Cities, China. 


\begin{tabular}{|c|c|c|}
\hline Characteristics & $\mathrm{N}$ & $\%$ \\
\hline Index cases of newly diagnosed men who have sex with men (MSM) & 446 & 100.0 \\
\hline Index cases recruited through self-testing at VCT sites & 435 & 97.5 \\
\hline Index cases recruited through partner tracing and HIV testing program & 11 & 2.5 \\
\hline \multicolumn{3}{|l|}{ Age of index case, years } \\
\hline $18-24$ & 108 & 24.2 \\
\hline $25-34$ & 200 & 44.8 \\
\hline $35-44$ & 78 & 17.5 \\
\hline 45 and above & 48 & 10.8 \\
\hline Missing & 12 & 2.7 \\
\hline \multicolumn{3}{|l|}{ Education level completed by index case } \\
\hline Primary school or below & 31 & 7.0 \\
\hline Junior middle school & 87 & 19.5 \\
\hline Senior high school & 104 & 23.3 \\
\hline College or above & 212 & 47.5 \\
\hline Missing & 12 & 2.7 \\
\hline \multicolumn{3}{|l|}{ Residence of index case } \\
\hline local county & 225 & 51.8 \\
\hline outside local county & 209 & 48.2 \\
\hline \multicolumn{3}{|l|}{ Number of sexual contacts disclosed by index case } \\
\hline 1 & 39 & 8.7 \\
\hline $2-5$ & 168 & 37.7 \\
\hline $6-10$ & 119 & 26.7 \\
\hline 11 and above & 120 & 26.9 \\
\hline \multicolumn{3}{|l|}{ Mode preference of index case, weighted ${ }^{\mathrm{a}}$} \\
\hline СHCT & 160 & 35.9 \\
\hline IDAPN & 126 & 28.3 \\
\hline HIVST & 13 & 2.9 \\
\hline Patient referral & 139 & 31.2 \\
\hline
\end{tabular}

MSM: men who have sex with men; CHCT: couples HIV testing and counselling; IDAPN: information-driven assisted partner notification; HIVST: assisted HIV self-testing;

a Weighted by assigning a preference value of one to each IC and proportionally assigning this value across his selection for each sexual contact 
Chaleatesistix contacts of newly diagnosed HIV-positive men who have sex with men enrolled in a partner tracing and HIWtesting program, Jrone 2014

through June 2016, in Hangzhou and Ningbo Cities, China. 


Characteristics $\quad \mathrm{N}$

Reachable sexual contacts reported by index cases

Age of sexual contacts, years

$$
17-24
$$

45 and above

Gender of sexual contacts

Male

Female

Missing

Current relationship status with sexual contacts, as disclosed by index case*

Type(s) of sexual relationship with sexual contacts, as disclosed by index case cases

Stable relationship with sexual contact

Stable, non-commercial same-sex relationship

\section{Non-Stable relationship with sexual contact}

Commercial same-sex relationship

Casual, non-commercial same-sex relationship

Other

Frequency of sexual contact as disclosed by index cases 


\begin{tabular}{|c|c|c|}
\hline Characteristics & & 9 \\
\hline Characteristics & $\mathrm{N}$ & $\%$ \\
\hline $3-4$ & 12 & 4.0 \\
\hline 5 and above & 0 & 0 \\
\hline Non-Stable relationship with sexual contact: total sexual contact & 244 & \\
\hline 1 & 76 & 31.1 \\
\hline 2 & 70 & 28.7 \\
\hline $3-4$ & 40 & 16.4 \\
\hline 5 and above & 33 & 13.5 \\
\hline \multicolumn{3}{|c|}{ Condom use in sexual contact between index case and sexual contact in the past 6 months, as disclosed } \\
\hline \multicolumn{3}{|l|}{ by index case } \\
\hline Never & 91 & 16.6 \\
\hline Inconsistent & 256 & 46.7 \\
\hline Consistent & 190 & 34.7 \\
\hline Missing & 11 & 2.0 \\
\hline
\end{tabular}

Table 3: HIV testing uptake and yield by modes of partner tracing and testing package among reachable sexual contacts of newly diagnosed HIV positive MSM in June 2014 through June 2016, in Hangzhou and Ningbo Cities, China.

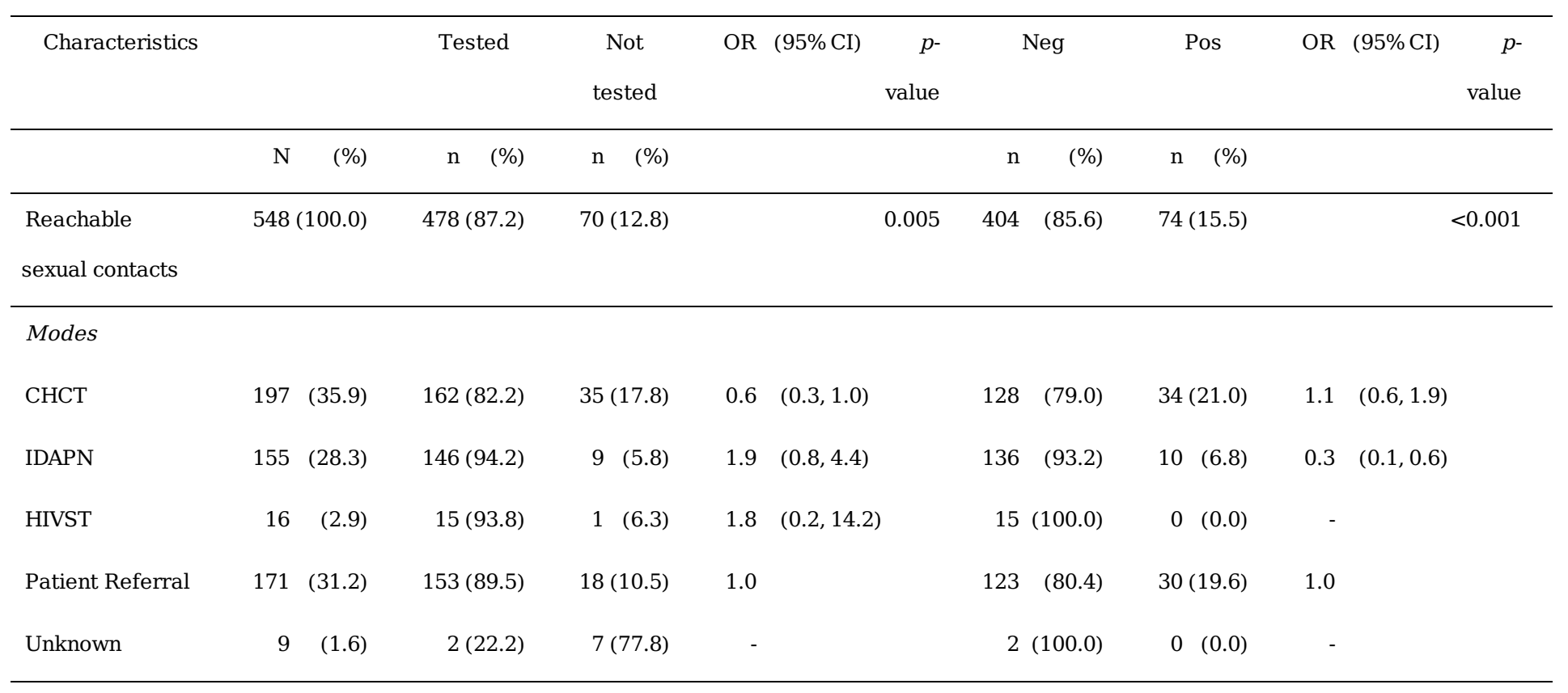

OR: Odds Ratio; CI: Confidence Interval; Neg: negative HIV test; Pos: positive HIV test; CHCT: couples HIV testing and counselling; IDAPN: Information-driven assisted partner notification; HIVST: assisted HIV self-testing 


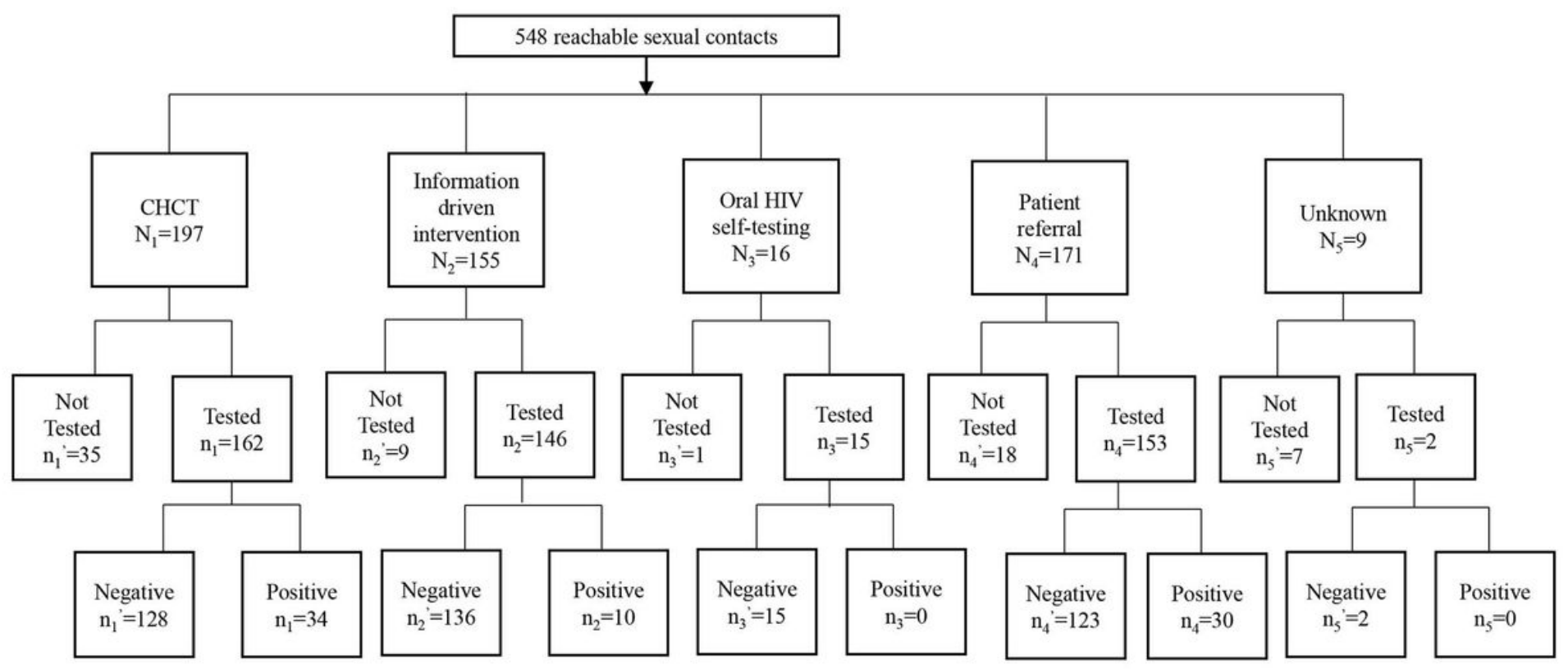

\section{Figure 1}

Cascade of partner tracing and HIV testing package among sexual contacts of newly diagnosed HIV positive men who have sex with men June 2014 through June 2016 in Hangzhou and Ningbo, China

\section{Supplementary Files}

This is a list of supplementary files associated with this preprint. Click to download.

- aditionalfile2.pdf

- aditionalfile3.pdf

- aditionalfile1.pdf 